\title{
Incidencia de la fibrosis quística en Paraguay
}

\author{
Incidence of cystic fibrosis in Paraguay
}

\author{
Marta Ascurra', Adriana Valenzuela', Mirna Salinas', Stella Rodríguez', Giovanna Porzio, \\ Lourdes Ortiz', Andrea Núñez', Pamela Alvarez
}

\section{RESUMEN}

Introducción: Fibrosis quística (FQ) patología genética, autosómica recesiva por mutaciones en el gen de la proteína reguladora de la conductancia transmembrana (CFTR cystic fibrosis transmembrane regulator). En una enfermedad multisistémica, afecta el aparato respiratorio, sistema digestivo, glándulas sudoríparas y conducto deferente. En Paraguay la detección, diagnóstico y tratamiento es obligatoria y gratuita para todo recién nacidos (RN). El tamizaje neonatal es realizado a través del dosaje de la tripsinainmuno reactiva (TIR) y la confirmación diagnóstica con el test del sudor. Objetivo: Reportar la incidencia de la FQ en la población de RN de Paraguay. Materiales y Métodos: Es un trabajo descriptivo, retrospectivo de corte trasversal, donde se analizaron los datos del Programa Nacional de Detección Neonatal (PNDN), de enero del 2015 a diciembre del 2017, para el análisis de la base de datos se utilizó una planilla Excel. Resultados: En el 2015 un 91\% (79.093/87.181) de las muestras ingresadas al Programa reunieron los criterios (edad y calidad de la muestra) para el estudio de la TIR, arrojando una incidencia de 1 en 6.591 RN. Para el 2016, esto correspondió a un 97\% (83.525/86.094) con una incidencia de 1 en 4.176 RN y por último en el 2017 , se tuvo un $97 \%$ (87.075/90.037) con una incidencia de 1 en 5.112 RN. Conclusión: La incidencia de la FQ en la población de recién nacidos del Paraguay, en los tres años que abarca este estudio, no presentan entre sí diferencias significativas, tampoco con las reportadas para la población hispánica.

Palabras claves: Incidencia, Fibrosis Quística, detección neonatal, Paraguay.

\section{ABSTRACT}

Introduction: Cystic fibrosis (CF), is an autosomal recessive genetic pathology that is caused by mutations in the transmembrane conductance regulatory protein (CFTR cystic fibrosis transmembrane regulator) gene. As a multisystem disease, it affects the respiratory system, digestive system, sweat glands and vas deferens. In Paraguay, detection, diagnosis and treatment is mandatory and free for all newborns (NB). Neonatal screening is performed by the immunoreactive trypsinogen assay (IRT) and diagnostic confirmation is performed by the sweat test. Objective: To report the incidence of CF in the NB population of Paraguay. Materials and Methods: This was a descriptive, retrospective, cross-sectional study, where data from the National Neonatal Screening Program (NNSP) were analyzed, from January 2015 to December 2017. To analyze the database, we used an Excel spreadsheet. Results: In 2015, 91\% (79,093 / 87,181) of the samples sent to the Program met the criteria (age and sample quality) for the IRT study, with an incidence of 1 in 6,591 NB. In $2016,97 \%(83,525 / 86,094)$ met criteria, with an incidence of 1 in 4,176 NB, while in 2017, 97\% $(87,075 / 90,037)$ met criteria, with an incidence of 1 in 5,112 NB. Conclusion: The incidence of CF in the newborn population of Paraguay, during the three years of this study, did not show significant differences between years, nor with those reported for the Hispanic population.

Keywords: Incidence, Cystic Fibrosis, neonatal detection, Paraguay.

\section{INTRODUCCIÓN}

La fibrosis quística (FQ) es una patología de origen genético de herencia autosómica recesiva, causada por mutaciones en el gen que codifica la proteína reguladora de la conductancia transmembrana:

\footnotetext{
${ }^{1}$ Ministerio de Salud Pública y Bienestar Social, Programa Nacional de Detección Neonatal. Asunción, Paraguay.

Correspondencia: Marta Ascurra; Correo: marta.ascurra@gmail.com

Conflicto de intereses: Los autores declaran no poseer conflicto de interés

Recibido: 03/08/2018; Aceptado: 28/12/2018

DOI: https://doi.org/10.31698/ped.46012019002
} 
Cystic fibrosis transmembrane conductance (CFTR, por sus siglas en inglés), que transporta activamente cloruro, bicarbonato y de manera secundaria agua. La condición causa alteraciones multisistémicas afectando principalmente al aparato respiratorio, el sistema digestivo, las glándulas sudoríparas y el conducto deferente ${ }^{(1,2)}$.

En Paraguay en el año 2003 se creó el Programa Nacional de Prevención de la Fibrosis Quística y del Retardo Mental. A partir del mismo se estableció la detección, diagnóstico y tratamiento de la FQ para todo recién nacido $(\mathrm{RN})$ de forma obligatoria y gratuita. En el año 2016 se cambió la primera denominación por Programa Nacional de Detección Neonatal $(\mathrm{PNDN})^{(3-5)}$.

Históricamente es importante recordar que a nivel mundial la inclusión de la FQ como una de las patologías a ser atendidas en los paneles de tamizaje neonatal ha sido un tema muy discutido, pues la misma no cumplía con requisitos esenciales para su inclusión, como por ejemplo contar con métodos de detección sensibles y tratamiento efectivo. Sin embargo con los avances en las técnicas de detección y los nuevos tratamientos esta patología fue incluida como parte de estos paneles. Estos factores influyeron en la decisión del PNDN de realizar, en sus inicios el tamizaje selectivo de FQ, a aquellos RN con síntomas o antecedentes familiares. Recién en el 2015 se logró dar cobertura a todas las muestras procedentes de las 18 Regiones Sanitarias (RS) del país ${ }^{(4 \cdot 6)}$.

La incidencia de la FQ así como su detección en una población, depende de factores tales como el ambiente, el grado de consanguineidad de la población estudiada y de las variaciones fenotípicas derivadas del tipo de mutación (grupo étnico). Todas ellas responsables de las diferencias observadas en la población mundial, ejemplos de estas variaciones son las reportadas por Australia, país en donde la incidencia es de 1 por cada $2.500 \mathrm{RN}$ mientras que en Japón se observa 1 caso por cada 100.000 a 350.000 RN. En Latinoamérica, Brasil presenta una incidencia de 1 en 1.600 en poblaciones con ascendencia europea y de 1 en $14.000 \mathrm{RN}$ en afrodescendientes, mientras que en Argentina se observa una incidencia de 1 en 6.573 nacidos vivos. Por otro lado, en México la incidencia es 1 en 8.500 y en el Caribe, en Cuba la incidencia reportada es de 1 en $3.900^{(7,8)}$. La presencia de descendientes de origen africano es importante en Ecuador, Colombia, Venezuela y Brasil, aunque su porcentaje de forma global no supere el 10\%. En Paraguay conforme a estudios genéticos la composición promedio del individuo paraguayo es la siguiente: $55,0 \%$ europea; $38,0 \%$ amerindia; $8,0 \%$ africana ${ }^{(4,79-11)}$.

El objetivo del presente estudio fue conocer la prevalencia de FQ durante los años 2015, 2016 y 2017 en la población de recién nacidos de 0 a 28 días de vida, del Paraguay, cuyas muestras fueron remitidas al Programa Nacional de Detección Neonatal para su estudio.

\section{METODOLOGÍA}

Este es un trabajo descriptivo, retrospectivo de corte trasversal. Se analizaron los resultados de las muestras de RN enviadas al PNDN de enero del 2015 a diciembre del 2017, provenientes de los 1.063 sitios de toma de muestra (STM), distribuidos en las 18 RS.

La recolección de las muestras de sangre total se llevó a cabo por punción del talón de los RN en papel de filtro Whatman 903, secadas a temperatura ambiente por 2 a 4 horas y refrigeradas hasta su remisión al Programa, entre los 2 y 10 días posteriores a su extracción.

Criterios de inclusión: en relación a la edad, se consideró toda muestra de RN hasta 28 días de vida y la calidad consistió en que la gota de sangre tenga un mínimo de $0,5 \mathrm{~cm}$ de diámetro, de distribución homogénea a ambos lados del papel.

La técnica utilizada en laboratorio consistió en la cuantificación de la tripsinainmunoreactiva (TIR), por el método de fluoroinmunoensayo en tiempo resuelto (DELFIA/PERKIN ELMER'S) en muestras de sangre seca recogidas en papel de filtro. El valor de referencia hasta $55 \mathrm{mgr} / \mathrm{dl}$.

Para la determinación de la positividad en el PNDN se utiliza la estrategia de trabajo $\operatorname{TIR}(+) / \operatorname{TIR}(+)$, que consiste en la realización de una primera prueba de la tripsinainmunoreactiva (TIR) y la solicitud de una segunda muestra a todos los casos que resultarán 
positivos en la primera. En caso de que ambas muestras presentaran resultados alterados, la confirmación diagnóstica se llevo a cabo mediante el test del sudor, que consiste en la medición del cloro por el método de titulación coulométrico en muestras de sudor. A los $\mathrm{RN}$ con edades superiores a los 30 días de vida al momento de la segunda muestra se les realizó de forma directa el test del sudor ${ }^{(12)}$.

Para el análisis de la base de datos se utilizó estadística descriptiva en una planilla Excel.

\section{RESULTADOS}

De enero del 2015 a diciembre del 2017 se recibieron 263.312 nuestras de recién nacidos, provenientes de los 1.063 sitios de toma de muestra distribuidos en las 18 RS, la distribución por año de acuerdo a las muestras colectadas, las procesadas, los porcentajes

Tabla 1. Número de muestras recibidas/estudiadas y casos de FQ detectados por año.

\begin{tabular}{ccccc}
\hline Año & $\begin{array}{c}\text { Muestras } \\
\text { Recibidas }\end{array}$ & $\begin{array}{c}\text { Muestras } \\
\text { Procesadas }\end{array}$ & $\begin{array}{c}\text { \% Muestras } \\
\text { analizadas }\end{array}$ & $\begin{array}{c}\text { Casos } \\
\text { Confirmados }\end{array}$ \\
\hline 2015 & 87.181 & 79.093 & 79.093 & 79.093 \\
2016 & 86.094 & 83.525 & 83.525 & 83.525 \\
\hline 2017 & 90.037 & 87.075 & 87.075 & 87.075 \\
\hline \multicolumn{7}{l}{ Fuente: Datos del PNDN (no publicados) }
\end{tabular}

de las mismas y los casos confirmados se observan en la Tabla 1.

Del este total de muestras analizadas en año 2015, 80 cumplieron la estrategia TIR/TIR, de las cuales en el 15\% (12/80) se confirmo FQ. Para el 2016, de 98 cumplieron la estrategia el 20,4\%(20/98) fueron confirmadas y finalmente en la año 2017 de los 120 que cumplieron la estrategia el 14\%(17/120) fueron confirmados. Es importante recalcar que 13 de los RN que tuvieron alterada la TIR en la primera determinación fallecieron Tabla 2.

En la Tabla 3, se presenta el resumen del № de nacimientos registrados por año, así como la sumatoria de estos. El número de STM, las muestras recibidas y analizadas en el periodo de tiempo del presente estudio y los casos confirmados de FQ, por RS.

Tabla 2. Distribución de casos confirmados de FQ e incidencia por año.

\begin{tabular}{|c|c|c|c|c|c|}
\hline Año & $\begin{array}{c}\text { IRT/IRT fuera } \\
\text { de rango }\end{array}$ & $\begin{array}{c}\text { Fallecidos } \\
\# \\
\end{array}$ & $\begin{array}{c}\text { Test del sudor } \\
\text { realizados* }\end{array}$ & Positivos & Incidencia \\
\hline 2015 & 80 & 2 & 120 & 12 & $1 \mid 6591$ \\
\hline 2016 & 98 & 6 & 152 & 20 & $1 \mid 4176$ \\
\hline 2017 & 120 & 5 & 173 & 17 & $1 \mid 5112$ \\
\hline TOTAL & 298 & 13 & 445 & 49 & \\
\hline
\end{tabular}

Tabla 3. Número de Nacimientos registrados-muestras recibidas/estudiadas y casos de FQ detectados por Región Sanitaria del 2015 al 2017.

\begin{tabular}{|c|c|c|c|c|c|c|c|c|}
\hline REGIÓN $\quad N^{\circ} \mathrm{D}$ & E NACIMIENTO & N DE NACIMIENTO & $\begin{array}{l}N^{\circ} \text { DE NACIMIENTO } \\
\text { REGISTRADOS } 2017\end{array}$ & $\begin{array}{l}N^{\circ} \text { DE NACIMIENTO } \\
\text { REGISTRADOS }\end{array}$ & $\begin{array}{l}N^{\circ} \mathrm{DE} \\
\text { STM* }\end{array}$ & $\begin{array}{l}\text { MUESTRAS } \\
\text { RECIBIDAS }\end{array}$ & $\begin{array}{l}\text { MUESTRAS } \\
\text { ESTUDIADAS }\end{array}$ & $\begin{array}{c}N^{\circ} \mathrm{DE} \\
\text { CASOS FQ }\end{array}$ \\
\hline & & REGISTRADOS 2016 & REGISTRADOS 2017 & $\begin{array}{c}\text { REGISTRADOS } \\
2015 \text { AL } 2017 \\
\end{array}$ & & RECIBIDAS & ESTUDIADAS & \\
\hline 1. Concepción & 5.071 & 4.551 & 5.315 & 14.937 & 68 & 10.362 & 9.394 & 3 \\
\hline 2. San Pedro & 9.126 & 8.484 & 8.385 & 25.995 & 75 & 15.318 & 14.425 & 0 \\
\hline 3. Cordillera & 5.051 & 4.593 & 4.699 & 14.343 & 69 & 9.943 & 9.9143 & 0 \\
\hline 4. Guaira & 3.265 & 3.097 & 2.922 & 9.284 & 68 & 7.479 & 7.479 & 3 \\
\hline 5. Caaguazu & 9.874 & 9.931 & 8.714 & 28.519 & 76 & 21.144 & 21.144 & 3 \\
\hline 6. Caazapa & 2.888 & 2.862 & 2.932 & 8.682 & 56 & 6.340 & 6.340 & 0 \\
\hline 7. Itapua & 7.483 & 8.818 & 8.037 & 24.338 & 90 & 18.646 & 18.646 & 2 \\
\hline 8. Misiones & 2.144 & 1.817 & 1.929 & 5.890 & 38 & 5.084 & 5.084 & 1 \\
\hline 9. Paraguari & 3.396 & 3.319 & 3.296 & 10.011 & 51 & 6.476 & 6.476 & 3 \\
\hline 10. Alto Paraná & 18.665 & 16.636 & 15.656 & 50.957 & 112 & 32.541 & 32.541 & 2 \\
\hline 11. Central & 35.542 & 34.906 & 36.824 & 107.272 & 124 & 73.417 & 73.417 & 20 \\
\hline 12. Ñeembucu & 974 & 984 & 922 & 2.880 & 60 & 2.412 & 2.412 & 0 \\
\hline 13. Amambay & 4.479 & 4.056 & 4.147 & 12.682 & 13 & 4.020 & 4.020 & 0 \\
\hline 14. Canindeyu & 4.916 & 4.304 & 5.772 & 14.992 & 62 & 8.346 & 8.346 & 0 \\
\hline 15. Pdte. Hayes & 2.582 & 2.244 & 3.092 & 7.918 & 36 & 3.269 & 3.269 & 0 \\
\hline 16. Boquerón & 1.442 & 1.526 & 1.451 & 4.419 & 18 & 3.444 & 3.444 & 0 \\
\hline 17. Alto Paraguay & 227 & 81 & 240 & 548 & 19 & 564 & 564 & 0 \\
\hline 18. Asunción & 9.724 & 9.514 & 9.166 & 28.404 & 28 & 34.507 & 34.507 & 12 \\
\hline $\begin{array}{l}\text { Departamento } \\
\text { /no reportado }\end{array}$ & 5.392 & 6.394 & 6.404 & 18.190 & 0 & 0 & 0 & 0 \\
\hline TOTAL & 132.241 & 128.117 & 129.903 & 390.261 & 1.063 & 263.312 & 249.693 & 49 \\
\hline
\end{tabular}

*STM = NÚMERO DW SITIOS DE TOMA DE MUESTRA. Fuente: http://www.dgeec.gov.py/ - Datos del PNDN (no publicados) 
Así también del total de nacimientos registrados a nivel país, durante estos tres años el porcentaje de cobertura para el estudio de la FQ aumento de un 60 a un $67 \%$ (Figura 1 ).

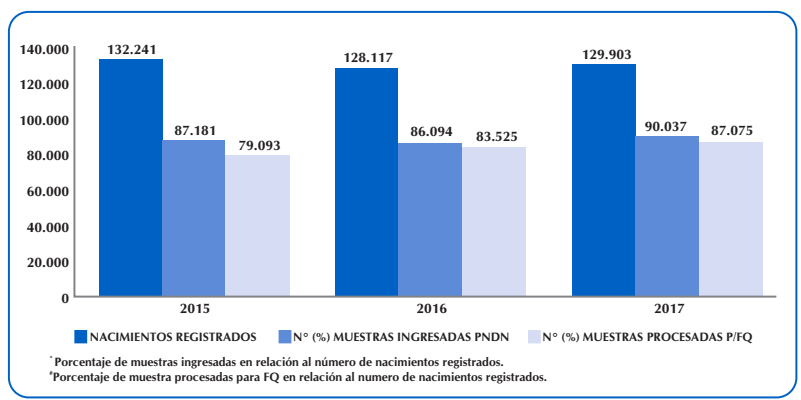

Figura 1. Comparativo de nacimientos registradosmuestras ingresadas y procesadas para FQ en el PNDN, periodo 2015-2017

\section{DISCUSIÓN}

La detección neonatal de la FQ en Paraguay ha sido garantizada por Ley desde el año 2003, la cobertura ha ido aumentando de forma paulatina, pudiéndose desde el 2015 contarse con los recursos suficientes no solo para absorber los costos del tratamiento de los pacientes diagnosticados con anterioridad y de los nuevos, sino para tamizar al $100 \%$ de las muestras recibidas, permitiéndonos el análisis de la incidencia de la patología en la población paraguaya de RN. En este trabajo se reporta por primera vez las incidencias anuales de la FQ en el Paraguay por tres años consecutivos, siendo estas similares a las descriptas en otros países sudamericanos, que oscilan entre $1 / 3000$ a $1 / 8500^{(8,11)}$.

La importancia de conocer la incidencia de FQ en nuestro país, no solo permite la proyección y el abastecimiento para la provisión en tiempo y forma de los medicamentos e insumos a ser entregados a los

\section{REFERENCIAS}

1. Kan S, Roth P, Piltcher O, Oliveira RD. Chronic rhinosinusitis and nasal polyposis in cystic fibrosis: update on diagnosis and treatment. J. Bras. Pneumol. 2015; 41(1):65-76. afectados, sino que además refuerza la importancia del diagnóstico precoz ante la evidencia demostrada en los resultados clínicos a corto y largo plazo en personas diagnosticadas por tamizaje neonatal ${ }^{(13)}$.

La mayor diferencia entre las muestras ingresadas y procesadas se observó durante el primer año de universalización de la pesquisa para $\mathrm{FQ}$, diferencia que disminuyó considerablemente los años siguientes, para lo cual se llevaron a cabo capacitaciones y envío de materiales informativos a los encargados de los STM.

La discrepancia observada entre el número de recién nacidos y el número de muestras recibidas correspondientes a la 18 RS (Asunción), podría deberse a diversos motivos como los partos domiciliaros estimados en poco más de 2.500 según el último reporte publicado por la Dirección General de Estadísticas Encuestas y Censos (DGEEC), y nacimientos que se produjeron en otra RS pero cuyas muestras fueron extraídas en STM de la capital, pasando a engrosar el número de muestras recibidas y estudiadas ${ }^{(14)}$.

Este estudio tiene la limitación de no conocerse las mutaciones genéticas de los pacientes, teniendo en cuenta los casos de atípicos de FQ que corresponden a mutaciones del CFTR que se presentan con pocos o ningún síntoma durante la niñez e incluso en la edad adulta ${ }^{(13,15)}$. Sin embargo, al ser el primer estudio epidemiológico sobre la incidencia de FQ en el país, aporta datos que hasta la fecha no se conocían sobre esta afección.

\section{CONCLUSIÓN}

La incidencia de la FQ en la población de recién nacidos del Paraguay en los tres años que abarca este estudio no presenta entre sí diferencias significativas ni con las reportadas para la población hispánica de la región. 
3. Paraguay. Ley N $N^{\circ}$ 5.732: De la Creación del Programa Nacional de Detección Neonatal y su ámbito de aplicación. 2016.

4. Mancuello Alum JN, Cabral de Bejarano MS. Sistema de Salud en Paraguay. Rev. Salud Pública Paraguay. 2011; 1(1):13-25.

5. MSPyBS. Guía Clínica: Fibrosis Quística. Asunción, Paraguay: Programa de Detección Neonatal, Ministerio de Salud Pública y Bienestar Social; 2009.

6. MSPyBS. Indicadores básicos de salud. [Página web]. Asunción, Paraguay: MSPyBS; 2018. [Citato 14 de enero del 2019]. Disponible en: http://portal.mspbs.gov.py/ digies/publicaciones/indicadores/

7. Robertson L. Cystic and Idiopathic Pulmonary Fibrosis: Risk Factors, Management and Long-term Health Outcomes. New York: Nova Science Publishers Inc; 2016.

8. Ribeiro Silva LV, Castaños C, Ruiz HH. Cystic fibrosis in Latin America-Improving the awareness. Journal of Cystic Fibrosis. 2016; 15(6):791-793.

9. PNUD, BM. Informe sobre desarrollo humano 2016: Desarrollo humano para todas las personas. Washington D.C., Estados Unidos: Communications Development Incorporated; 2016.
10. Collazo T, Magarino C, Chavez R, Suardiaz B, Gispert $S$, Gomez M. et al. Frequency of delta-F508 mutation and XV2C/KM19 haplotypes in Cuban cystic fibrosis families. Hum. Hered. 1995; 45:55-57.

11. Hernandez E, Carnevale A. Spectrum of CFTR mutations in Mexican cystic fibrosis patients: identification of five novel mutations (W1098C, 846delT, P750L, 4160insGGGG and 297-1G-->A). Hum. Genet. 2000; 106:360-365.

12. Borrajo GJC. Pesquisa neonatal de enfermedades congénitas. [Tesis Doctoral]. Universidad Nacional de la Plata, La Plata: Facultad de Ciencias Exactas; 2011.

13. Ibarra González I, Gutiérrez Morales G, Vela Amieva M, Castillo Mogel JA, Herrera Pérez LA, Caamal Parra G, et al. Retos y oportunidades en la implementación del tamiz neonatal para fibrosis quística. Acta Pediatr Mex. 2018; SI(39):35S-46S

14. OPS, OMS. Indicadores básicos de salud. [Página web]. Asunción, Paraguay: OPS; 2018. [Citado el 11 de enero 2019]. Disponible en: https://www.paho.org/par/index. php?option=com_content\&view=article\&id=25:indicador es-basicos-salud\&Itemid=135.

15. Dodge JA. A millennial view of cystic fibrosis. Dev Period Med. 2015; 19(1):9-13. 